\title{
Associations between perceived environmental pollution and health-related quality of life in a Chinese adult population
}

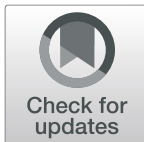

Bingxue $\operatorname{Han}^{1,2,3,4}$ (D)

\begin{abstract}
Background: Health-related quality of life (HRQoL) measures are being used in increasingly diverse populations. However, there have no known studies to date to examine the associations between perceived environmental pollution and HRQoL in a Chinese population. This study aimed to report the associations between air, water, noise pollution and HRQoL among Chinese adult population.

Methods: A cross-sectional survey data was used from East Asian Social Survey 2010 with a sample of 3866 Chinese populations regarding environmental pollution. HRQoL was computed by SF-12 and reflected by physical and mental component summary score (PCS \& MCS). Ordinary least regression analyses were used to examine associations between perceived environmental pollution and PCS and MCS scores. Models in SPSS PROCESS were selected to demonstrate the moderating and mediating effects.

Results: Only considering one pollutant, perceived air pollution and perceived water pollution had significant associations with PCS and MCS scores. Perceived noise pollution had significant associations with PCS scores.

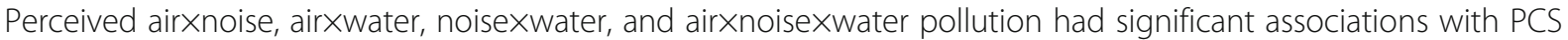
and MCS scores. Conditional (moderated) mediation showed that there were no moderating effects and mediating effects of perceived one pollutant on another pollutant.
\end{abstract}

Conclusions: Co-occurring perceived environmental pollution were mainly associated with progressive increase in PCS and MCS scores among the Chinese adult population. These results suggested that some effective policies should be carried out to improve environmental quality in Chinese adult population.

Keywords: Perceived environmental pollution, Health-related quality of life, PCS scores, MCS scores, Chinese adult population

\section{Introduction}

A number of studies indicated that air pollution possibly worsened the health status of patients. For example, a study conducted in peri-urban Peru concluded higher traffic air pollution exposure was associated with worse rhinitis QoL among asthmatic children [1]. A small cohort study of

Correspondence: hanbingxue0451@163.com

${ }^{1}$ International Issues Center, Xuchang University, Xuchang, Henan, China

${ }^{2}$ Family Issues Center, Xuchang University, Xuchang, Henan, China

Full list of author information is available at the end of the article patients with fibrotic sarcoidosis indicated that PM2.5 exposure was associated with increased severity of respiratory and quality of life symptoms [2]. A field study suggest that PM, $\mathrm{NO}_{2}$, and $\mathrm{O}_{3}$ cause respiratory symptoms leading to HRQoL deterioration [3]. Regarding asthma, a study concluded that minimizing exposure to air pollution might improve asthmatic patients' disease management and HRQoL [4]. The molecular mechanisms of susceptibility were also reported [5]. Clinically, air pollution produced detrimental effects on mental health by various components of air pollution

C C The Author(s). 2020 Open Access This article is licensed under a Creative Commons Attribution 4.0 International License, which permits use, sharing, adaptation, distribution and reproduction in any medium or format, as long as you give appropriate credit to the original author(s) and the source, provide a link to the Creative Commons licence, and indicate if changes were made. The images or other third party material in this article are included in the article's Creative Commons licence, unless indicated otherwise in a credit line to the material. If material is not included in the article's Creative Commons licence and your intended use is not permitted by statutory regulation or exceeds the permitted use, you will need to obtain permission directly from the copyright holder. To view a copy of this licence, visit http://creativecommons.org/licenses/by/4.0/ The Creative Commons Public Domain Dedication waiver (http://creativecommons.org/publicdomain/zero/1.0/) applies to the data made available in this article, unless otherwise stated in a credit line to the data. 
translocated central nervous system [6]. Mentally, PM2.5 was associated with depressive and anxiety symptoms, with associations the strongest among individuals with lower socioeconomic status [7]. A cross-sectional study in two slums of Nairobi indicated that perceived air pollution level was positively associated with perceived health risks [8]. The deleterious effects of air pollution on human health had been consistently documented by many epidemiologic studies worldwide among the general public [9-13]. Exposure to air pollution had been confirmed to reduce enthusiasm of participation in outdoor physical activity $[14,15]$. But, the limited information was investigated in China.

A substantial literature showed water pollution impaired the health status substantially. Previous research has established that polluted water caused by economic growth is one the main risks of public health in mainland China [16-18]. There is a growing body of literature that recognizes the role of water pollution in morbidity and mortality in international community [19, 20]. Also, the generalisability of much published research that disease prevalence and mortality were remarkably associated with water pollution is confirmed in China's context [21]. A number of studies indirectly reflect the association between water pollution and health outcomes, but there was little literature reported the relationship between water pollution and HRQoL. Water pollution was confirmed to be a major risk factor for the past and potential health deterioration. A study in Saudi Arabia highlighted the urgent need for monitoring and controlling wastewater discharge in Wadi Hanifa to ensure public safety [22]. A systematic review concluded consumption of toxicant tainted fishes in deteriorating Ganga water might cause serious illness including cancer [23]. In Bangladesh, water pollution was crucial to evaluate public health risk, particularly among children [24]. A study in The Huai River basin located in eastern China indicated that the current esophageal cancer mortality rate was mainly caused by water pollution from the previous 8 years [25]. But, the truth on the relationship between water pollution and quality of life has not been discovered in Chinese settings.

There was full of literature that addressed the intersection of quality of life and noise pollution worldwide. For instance, a cross-sectional questionnaire-based study shows that individuals with high noise sensitivity (NS) tend to have degraded HRQOL compared to individuals with low NS [26]. A cross-sectional study indicated that noise sensitivity appear to degrade the quality of life of those with a mild traumatic brain injury [27]. A systematic review found there was lack of evidence for noise effects across studies for many of the quality of life [28]. Noise pollution was pervasive even in U.S. protected areas [29]. Workplace health hazard of noise pollution were documented in factories [30], metalwork and woodwork industries [31], and traffic [32]. Methodologically, literature review and empirical studies were adopted to discuss the relationship between noise pollution and quality of life in the international academic circle [33, 34]. In western countries, environmental noise produced by airport caused psychological and physiological disorders [35, 36]. Association between noise pollution and some specific diseases were reported [37, 38]. Strategically, various countries and international organizations were making efforts to eliminate adverse public health due to environmental noise pollution [39]. But to date, there is little knowledge of the relationship revealed in mainland China.

Several prior studies used SF-12 to measure HRQoL due to its scientific and comprehensive nature [40, 41]. In this study, SF-12 was used to evaluate the associations between three pollutions and HRQoL in a Chinese adult population. Regarding the psychometric properties, several studies have reported the SF-12 was translated and confirmed to be a reliable and valid measure of HRQoL in a range of medical conditions [42], as well as in the general population [43]. Also, the standard SF-12 was valid and equivalent to measure HRQoL for the Chinese [44].

Since the first year in the new century, especially from 2008 to 2012, environmental pollution has become the primary problem restricting economic development and impacting health status among the public in China. Thus, it could be speculated that air, water, and noise pollution had become substantial environmental issues affecting health status and HRQoL. Here, this study adopted SF-12 to explore the associations between perceived environmental pollution and HRQoL in a Chinese adult population. Here, HRQoL was expressed by physical and mental component summary score (PCS \& MCS) after the calculation of SF-12. The co-occurring perceived environmental pollution was constructed in the main multiple research scenarios. Interactions between perceived environmental pollution would be analyzed in the scenarios.

\section{Methods}

\section{Data source}

This study used cross-sectional survey data (East Asian Social Survey, http://www.eassda.org/) from a three stage PPS of all Chinese aged 18 and above. This crosssectional survey data were derived from the China Comprehensive Social Survey (CGSS 2010), which was jointly conducted by Renmin University of China and relevant academic institutions. Fieldwork Dates were from July to December, 2010. Its initial sample size was 5370. But, a valid response rate was $71.99 \%$. After the respondents in Japan, South Korea, and Taiwan were deleted, the China sample size was 3866 . 
The sampling design made in CGSS 2010 was multistage stratified design (http://cgss.ruc.edu.cn/index.php?r= index/sample). There were 3 sampling stages: primary sampling units (PSUs) were county-level units, there were 2762 PSUs in the sampling frame; secondary sampling units (SSUs) were community-level units; 25 households in third-level sampling units were sampled with PPS method in selected SSUs; 18 and above adult were sampled with Kish grid in each selected household. The total sample size of 2010 design was 12,000 households and 2000 were in self-representative stratum.

The questionnaire was mainly composed of health status, health behavior, medical care, medical insurance/social security insurance, alternative medicine, social support/ social trust, environment, epidemiology, family care need and care management, worries about ageing, addiction, and body shape. The data were collected by face-to-face interviewing.

\section{Dependent variable}

The Physical Functioning (PF), Role Physical (RP), Bodily Pain (BP), General Health (GH), Vitality (VT), Social Functioning (SF), Role Emotional (RE), Mental Health $(\mathrm{MH})$, Physical and Mental Composite Scores (PCS \& MCS) were calculated with the sf12 program (Bruun, 2015; https://ideas.repec.org/c/boc/bocode/s458125.html) [45]. A higher score in the respective summary scales represent a higher level of functioning. Their mean score (SD), Floor (\%), and Ceiling (\%) could be seen in Table 1. Thus, mean MCS-12 score was lower than the mean PCS12 score of the sample.

\section{Main variables}

The sociodemographic factors that were of interest to this study included age (years), sex $(0=$ female, $1=$ male $)$, marital status, religious status, community type $(1=\mathrm{a}$ big city, 2 = the suburbs or outskirts of a big city, $3=a$ town or a small city, and $4=$ a country village), years of schooling, body mass index, employment relationship, number of household members, total household income (Chinese Yuan), and chronic conditions $(1=$ with, $0=$ without). The marital status included married $(=1)$ and unmarried $(=0$, widowed, divorced, separated (have intention to divorce), never married, and cohabiting). The religious status included "no religion" $(=1)$ and "religion" (=0, Roman Catholic, Protestant, Islam, Buddhism, and other eastern religions). The variable, employment relationship, was categorized into employment (employee, self-employed without employees, self-employed with employees, and working for own family's business) coded 0 and unemployment (having no current work income) coded 1. Frequencies of alcoholic drinks was divided by yes $(1=$ daily, several times a week, several times a month, and several times a year or less often) and no $(0=$ never $)$. Frequencies of smoking was divided by yes $(1=$ daily, several times a week, several times a month, and several times a year or less often) and no $(0=$ never $)$.

Perceived air/noise/water pollution was assessed by the questions: "How severe is air pollution in the area of respondents' local residence", "How severe is noise pollution in the area of respondents' local residence", and "How severe is water pollution in the area of respondents' local residence". Their response options were very severe $(=1)$, somewhat severe $(=2)$, not so severe $(=3)$, and not severe at all $(=4)$ and recoded as yes $(1=$ very severe and somewhat severe $)$ and no $(0=$ somewhat severe and not so severe).

\section{Statistical methods}

In the dataset, the response options with "tw: not asked", "dk, refused", and "undisclosed" were cleaned as missing values. Descriptive statistics for the sample were calculated on the basis of gender difference. Similarly, air, water, and noise pollution were dichotomized into binary values $(1=$ very severe and somewhat severe, $0=$ not so severe and not severe at all). Thus, four binary variables, including air $\times$ water, noise $\times$ water, air $\times$ noise, and

Table 1 Mean score (SD), Floor (\%), and Ceiling (\%) for 8 domains of SF-12 (total=3866)

\begin{tabular}{|c|c|c|c|c|c|c|}
\hline & Observations & Mean score & SD & Floor (\%) & Ceiling (\%) & Completion rates \\
\hline $\mathrm{PF}$ & 3847 & 50.540 & 9.724 & 4.86 & 66.36 & 99.51 \\
\hline $\mathrm{RP}$ & 3842 & 47.770 & 9.832 & 2.06 & 33.13 & 99.38 \\
\hline $\mathrm{BP}$ & 3848 & 47.382 & 12.471 & 4.76 & 49.32 & 99.53 \\
\hline $\mathrm{GH}$ & 3861 & 49.377 & 12.276 & 4.14 & 24.71 & 99.87 \\
\hline VT & 3859 & 54.673 & 10.732 & 2.54 & 24.64 & 99.82 \\
\hline SF & 3849 & 46.530 & 10.663 & 3.04 & 40.11 & 99.56 \\
\hline $\mathrm{RE}$ & 3859 & 45.164 & 9.978 & 0.73 & 30.97 & 99.82 \\
\hline $\mathrm{MH}$ & 3847 & 50.877 & 10.058 & 0.10 & 15.34 & 99.51 \\
\hline PCS & 3781 & 49.415 & 10.939 & 0.03 & 0.03 & 97.80 \\
\hline MCS & 3781 & 48.914 & 9.357 & 0.03 & 0.03 & 97.80 \\
\hline
\end{tabular}


air $\times$ noise $\times$ water were produced in order to reflect coexisting pollution. These binary variables were valued as 1 if co-occurring pollution existed and as 0 if not. For convenience, the main variables, the abbreviated in the parentheses of marital status (maritaln), religious status (religion), community type (urbrural), years of schooling (educyrs), body mass index (bmi), number of household members (hhdnum), total household income ( $c n$ _hinc), and chronic conditions (chronic), frequencies of alcoholic drinks (alcoholicg), and frequencies of smoking (smokingg) were adopted in the regression analyses.

Considering confounding factors in a stepwise fashion, the associations between potential covariates and PCS and MCS scores were assessed using linear regression models. The chest command in Stata was used to examine confounding effects for perceived air pollution, water pollution, noise pollution, air $\times$ water pollution, air $\times$ noise pollution, noise $\times$ water pollution, and air $\times$ noise $\times$ water pollution separately. The socioeconomic factors, lifestyle, and health status were considered as potential confounding variables. When screening removed variables, change-in-estimate criterion with a $0.09 \%$ cutoff was adopted [46, 47].

Controlling for the covariates in the linear regression models, moderation and mediation analysis would be performed to reflect the association between perceived environmental pollution and HRQoL. The effect of one pollutant on PCS and MCS scores could be moderated another two pollutants. There was no current literature to support one pollutant did a harm through another pollutant. Thus, when air pollutant was conceived as independent variables, noise and water pollutants were considered as hypothesized moderators rather than mediators. Thus, the moderation could be performed by moderation models one by one in AF Hayes Process procedure for SPSS Release 2.13 (www.afhayes.com; www.guilford.com/p/hayes3) and tested in the simple moderation models 1,2 , and 3 . If the statistical outcomes could not reach satisfied significance, some moderated mediation models would be explored in the other complex models. One of PCS and MCS scores could be mediator, the other could be outcome variable. If one pollutant could be independent variable, the other two pollutants could be moderators.

The dependent variables were constructed with PCS and MCS scores. All data were analyzed using the weighted survey procedure in Stata 14.0.

\section{Results}

\section{Descriptive analysis}

Table 2 summarized the demographic characteristics of respondents. Respondents were predominantly female (51.58\%), aged $36-58$ years $(51.54 \%)$, married $(79.57 \%)$, religionless (87.18\%), urban (62.47\%), employed (64.25\%), without chronic conditions (66.01\%), without alcoholic drinks (69.20\%), without smoking (62.20\%), without perceived air pollution (70.96\%), without perceived noise pollution $(70.10 \%)$, without perceived water pollution (73.57\%), without perceived air $\times$ noise pollution $(81.40 \%)$, without perceived air $\times$ water pollution $(82.11 \%)$, without perceived noisexwater pollution $(88.83 \%)$, and without perceived air $\times$ noise $\times$ water pollution $(87.44 \%)$.

Considering gender difference, there were significant differences in religion group, community type, years of schooling, body mass index, employment relationship, chronic condition, frequencies of alcoholic drinks, and frequencies of smoking.

\section{Regression analysis}

Associations of PCS and MCS scores with sociodemographic factors, lifestyle, and perceived environmental pollution were conducted with ordinary least regressions.

\section{Single perceived environmental pollution}

Supplementary Table 1 showed that the change-inestimates of hhdnum $(=0.02 \%)$ and smokingg $(=0.01 \%)$ were lower than the $0.09 \%$ cutoff criterion, which indicated they were covariates. However, the change-inestimates of age $(=3.30 \%)$, sex $(=0.83 \%)$, maritaln $(=$ $1.78 \%)$, religion $(=0.13 \%)$, urbruraln $(=0.70 \%)$, educyrs $(=$ $180.00 \%)$, bmi $(=6.45 \%)$, employment $(=0.80 \%)$, cn_hinc $(=0.31 \%)$, chronic $(=36.13 \%)$, and alcoholicg $(=0.19 \%)$ were higher than the $0.13 \%$ cutoff criterion, which indicated they were potential confounding variables.

Supplementary Table 2 showed that the change-inestimates of smokingg $(=0.05 \%)$ was lower than the $0.09 \%$ cutoff criterion, which indicated they were covariates. However, the change-in-estimates of age $(=0.5098 \%)$, sex $(=3.2276 \%)$, maritaln $(=1.16 \%)$, religion $(=0.28 \%)$, urbruraln (=69.33\%), educyrs (=11.03\%), bmi (=8.11\%), employment (=0.4911\%), hhdnum (=0.68\%), cn_hinc (=1.94\%), chronic $(=10.91 \%)$, and alcoholicg $(=0.16 \%)$ were higher than the $0.15 \%$ cutoff criterion, which indicated they were potential confounding variables.

Supplementary Table 3 showed that the change-inestimates of bmi $(=0.00 \%)$ and hhdnum (=0.02\%) were lower than the $0.09 \%$ cutoff criterion, which indicated they were covariates. However, the change-in-estimates of age $(=1.3050 \%)$, sex $(=0.6354 \%)$, maritaln $(=0.48 \%)$, religion $(=$ $0.10 \%)$, urbruraln $(=4.3993 \%)$, educyrs $(=362.3407 \%)$, employment $(=4.82 \%)$, cn_hinc $(=0.09 \%)$, chronic $(=47.0650 \%)$, alcoholicg $(=0.4049 \%)$, and smokingg $(=3.6444 \%)$ were higher than the $0.09 \%$ cutoff criterion, which indicated they were potential confounding variables.

Supplementary Table 4 showed that the change-inestimates of bmi $(=0.0692 \%)$ and smoking $(=0.0508 \%)$ was lower than the $0.09 \%$ cutoff criterion, which indicated they were covariates. However, the change-in- 
Table 2 Sample characteristics by gender

\begin{tabular}{|c|c|c|c|c|c|}
\hline Participant characteristics & Female & Male & Total & Ch2 & $P$ value \\
\hline Age (Years), Median (IQR) & $46(36-58)$ & $47(36-59)$ & $46(36-58)$ & 86.4658 & 0.152 \\
\hline Marital Status & & & & 0.6203 & 0.431 \\
\hline Unmarried, N (\%) & $415(10.79)$ & $371(9.64)$ & & & \\
\hline Married, N (\%) & $1568(40.76)$ & $1493(38.81)$ & & & \\
\hline Religion & & & & 18.1988 & $0.000^{* * *}$ \\
\hline Religionless, N (\%) & $1688(43.81)$ & $1671(43.37)$ & & & \\
\hline Religious, N (\%) & $299(7.76)$ & $195(5.06)$ & & & \\
\hline Community Type & & & & 3.0775 & $0.079^{*}$ \\
\hline Urban, N (\%) & $1272(32.90)$ & $1143(29.57)$ & & & \\
\hline Rural, N (\%) & $722(18.68)$ & $729(18.86)$ & & & \\
\hline Years of schooling, Median (IQR) & $9(6-11)$ & $9(6-12)$ & $9(6-12)$ & 127.5275 & $0.000^{* * *}$ \\
\hline BMI, Median (IQR) & $21.64(19.56-24.34)$ & $22.49(20.52-24.80)$ & $22.04(20.03-24.61)$ & $2.2 e+03$ & $0.000^{* * *}$ \\
\hline Employment & & & & 90.2070 & $0.000^{* * *}$ \\
\hline Employed, N (\%) & $1129(29.57)$ & $1324(34.68)$ & & & \\
\hline Unemployed, N (\%) & $847(22.18)$ & $518(13.57)$ & & & \\
\hline Number of household members, Median (IQR) & $2(2-3)$ & $2(2-3)$ & $2(2-3)$ & 13.7062 & 0.133 \\
\hline Total household income, Median (IQR) & $24,000(12000-45,000)$ & $24,000(12000-45,600)$ & $24,000(12000-45,000)$ & 544.1617 & 0.609 \\
\hline Chronic Condition & & & & 14.6145 & $0.000^{* * *}$ \\
\hline Without, N (\%) & $1260(32.59)$ & $1292(33.42)$ & & & \\
\hline With, N (\%) & $734(18.99)$ & $580(15.00)$ & & & \\
\hline Alcoholic drinks & & & & $1.3 e+03$ & $0.000^{* * *}$ \\
\hline No, N (\%) & $1891(49.23)$ & $767(19.97)$ & & & \\
\hline Yes, N (\%) & $84(2.19)$ & $1099(28.61)$ & & & \\
\hline Smoked & & & & $1.0 e+03$ & $0.000^{* * *}$ \\
\hline No, N (\%) & $1706(44.49)$ & $679(17.71)$ & & & \\
\hline Yes, N (\%) & $267(6.96)$ & $1183(30.85)$ & & & \\
\hline Air pollution & & & & 1.0092 & 0.315 \\
\hline No, N (\%) & $1395(36.18)$ & $1341(34.78)$ & & & \\
\hline Yes, N (\%) & $591(15.33)$ & $529(13.72)$ & & & \\
\hline Noise pollution & & & & 0.5241 & 0.469 \\
\hline No, N (\%) & $1404(36.40)$ & $1300(33.70)$ & & & \\
\hline Yes, N (\%) & $584(15.14)$ & $569(14.75)$ & & 0.1518 & 0.697 \\
\hline \multicolumn{6}{|l|}{ Water pollution } \\
\hline No, N (\%) & $1465(38.03)$ & $1369(35.54)$ & & & \\
\hline Yes, N (\%) & $519(13.47)$ & $499(12.95)$ & & & \\
\hline Air×noise pollution & & & & 0.3983 & 0.528 \\
\hline No, N (\%) & $1609(41.74)$ & $1529(39.66)$ & & & \\
\hline Yes, N (\%) & 377 (9.78) & $340(8.82)$ & & & \\
\hline Air×water pollution & & & & 0.1151 & 0.734 \\
\hline No, N (\%) & $1625(42.20)$ & $1537(39.91)$ & & & \\
\hline Yes, N (\%) & $359(9.32)$ & $330(8.57)$ & & & \\
\hline Noisexwater pollution & & & & 0.3881 & 0.533 \\
\hline No, N (\%) & $1684(43.73)$ & $1598(41.50)$ & & & \\
\hline Yes, N (\%) & $300(7.79)$ & $269(6.99)$ & & & \\
\hline
\end{tabular}


Table 2 Sample characteristics by gender (Continued)

\begin{tabular}{|c|c|c|c|c|c|}
\hline Participant characteristics & Female & Male & Total & Ch2 & $P$ value \\
\hline Airxnoisexwater pollution & & & & 0.0663 & 0.797 \\
\hline No, N (\%) & $1732(44.98)$ & $1635(42.46)$ & & & \\
\hline Yes, N (\%) & $252(6.54)$ & $232(6.02)$ & & & \\
\hline
\end{tabular}

Note: ***, ** and ${ }^{*}$ indicates 1,5 and $10 \%$ significance level, respectively

estimates of age $(=1.2981 \%)$, sex $(=4.3169 \%)$, maritaln $(=$ $0.0974 \%)$, religion $(=0.5219 \%)$, urbruraln $(=25.0116 \%)$, educyrs $(=108.2362 \%)$, employment $(=1.1174 \%)$, hhdnum $(=0.2426 \%)$, cn_hinc $(=0.6419 \%)$, chronic $(=21.1382 \%)$, and alcoholicg $(=0.2316 \%)$ were higher than the $0.09 \%$ cutoff criterion, which indicated they were potential confounding variables.

Supplementary Table 5 showed that the change-inestimates of religion $(=0.05 \%)$ was lower than the $0.09 \%$ cutoff criterion, which indicated they were covariates. However, the change-in-estimates of age $(=2.61 \%)$, sex $(=1.03 \%)$, maritaln $(=1.99 \%)$, urbruraln $(=32.76 \%)$, educyrs $(=6.03 \%)$, bmi $(=1.99 \%)$, employment $(=6.26 \%)$, hhdnum (=0.14\%), cn_hinc $(=0.28 \%)$, chronic $(=20.87 \%)$, alcoholicg $(=0.15 \%)$, and smoking $(=0.99 \%)$ were higher than the $0.14 \%$ cutoff criterion, which indicated they were potential confounding variables.

Supplementary Table 6 showed that change-inestimates of religion $(=0.02 \%)$ was lower than the $0.09 \%$ cutoff criterion, which indicated they were covariates. However, the change-in-estimates of age $(=1.03 \%)$, sex $(=4.30 \%)$, maritaln $(=2.79 \%)$, urbruraln $(=22.92 \%)$, educyrs (=1.49\%), bmi (=1.81\%), employment (=1.08\%), hhdnum (=2.8543\%), cn_hinc $(=2.61 \%)$, chronic $(=9.17 \%)$, alcoholicg $(=0.10 \%)$, and smoking $(=0.24 \%)$ were higher than the $0.09 \%$ cutoff criterion, which indicated they were potential confounding variables.

Table 3 showed the associations between one perceived environmental pollution and QoL. Only considering air pollution, perceived air pollution had significant associations with PCS scores $(\beta=11.442,95 \% \mathrm{CI}$ : 9.589, 13.295) and MCS scores $(\beta=32.329,95 \% \mathrm{CI}: 30.924$, $33.734)$, perceived noise pollution had significant associations with PCS scores $(\beta=1.670,95 \% \mathrm{CI}$ : $0.649,2.691)$, perceived water pollution had significant associations with PCS scores $(\beta=44.099,95 \% \mathrm{CI}$ : 42.918,45.281) and MCS scores $(\beta=42.561,95 \% C I$ : 41.454,43.669).

Hhdnum had significant associations with PCS scores $(\beta=10.738,95 \% \mathrm{CI}: 9.951,11.524)$ in air polluted population and PCS scores $(\beta=1.719,95 \% \mathrm{CI}: 1.234,2.204)$ in the population with perceived noise pollution. Smokingg had significant associations with PCS scores $(\beta=17.064$, 95\%CI: $14.577,19.551)$ and MCS scores $(\beta=40.310$, $95 \% \mathrm{CI}: 39.333,41.287)$ in the population with perceived air pollution, MCS scores $(\beta=1.818,95 \% \mathrm{CI}$ : 0.979 , $2.658)$ in the population with perceived noise pollution. $B m i$ had significant associations with PCS scores $(\beta=$ 1.945, 95\%CI: $1.882,2.008)$ and MCS scores $(\beta=2.115$,

Table 3 Associations between one perceived environmental pollution and PCS and MCS scores, Coefficients (95\%Cl)

\begin{tabular}{|c|c|c|c|c|c|c|}
\hline & \multicolumn{2}{|l|}{ Air pollution } & \multicolumn{2}{|c|}{ Noise pollution } & \multicolumn{2}{|l|}{ Water pollution } \\
\hline & PCS scores & MCS scores & PCS scores & MCS scores & PCS scores & MCS scores \\
\hline Air pollution & $\begin{array}{l}11.442 * * * \\
(9.589,13.295)\end{array}$ & $\begin{array}{l}32.329 * * * \\
(30.924,33.734)\end{array}$ & & & & \\
\hline Noise pollution & & & $\begin{array}{l}1.670^{* * *} \\
(0.649,2.691)\end{array}$ & $\begin{array}{l}-0.069 \\
(-0.981,0.842)\end{array}$ & & \\
\hline Water pollution & & & & & $\begin{array}{l}44.099^{* * *} \\
(42.918,45.281)\end{array}$ & $\begin{array}{l}42.561^{* * *} \\
(41.454,43.669)\end{array}$ \\
\hline Hhdnum & $\begin{array}{l}10.738^{* * *} \\
(9.951,11.524)\end{array}$ & & $\begin{array}{l}1.719^{* * *} \\
(1.234,2.204)\end{array}$ & & & \\
\hline Smokingg & $\begin{array}{l}17.064^{* * *} \\
(14.577,19.551)\end{array}$ & $\begin{array}{l}40.310^{* * *} \\
(39.333,41.287)\end{array}$ & & $\begin{array}{l}1.818^{* * *} \\
(0.979,2.658)\end{array}$ & & \\
\hline BMI & & & $\begin{array}{l}1.945^{* * *} \\
(1.882,2.008)\end{array}$ & $\begin{array}{l}2.115^{* * *} \\
(2.088,2.143)\end{array}$ & & \\
\hline Religion & & & & & $\begin{array}{l}35.643^{* * *} \\
(33.246,38.041)\end{array}$ & $\begin{array}{l}35.760^{* * *} \\
(33.459,38.061)\end{array}$ \\
\hline R-squared & 0.823 & 0.478 & 0.942 & 0.949 & 0.313 & 0.317 \\
\hline Number of observation & 3750 & 3751 & 3771 & 3748 & 3763 & 3763 \\
\hline
\end{tabular}

Note: ${ }^{* * * *}$ and ${ }^{*}$ indicates 1,5 and $10 \%$ significance level, respectively. Hhdnum $=$ number of household members. Smokingg $=$ frequencies of smoking. Religion $=$ religious status 
95\%CI: 2.088, 2.143) in the population with perceived noise pollution. Religion had significant associations with PCS scores ( $\beta=35.643,95 \%$ CI: 33.246, 38.041) and MCS scores $(\beta=35.760,95 \% \mathrm{CI}$ : $33.459,38.061)$ in the population with perceived water pollution.

\section{Two co-occurring perceived environmental pollution}

Supplementary Table 7 showed that the change-inestimates of hhdnum $(=0.04 \%)$ were lower than the $0.09 \%$ cutoff criterion, which indicated they were covariates. However, the change-in-estimates of age $(=0.73 \%)$, sex $(=0.30 \%)$, maritaln $(=0.47 \%)$, religion $(=0.13 \%)$, urbruraln $(=1.31 \%)$, educyrs $(=286.62 \%)$, bmi $(=7.64 \%)$, employment $(=0.84 \%)$, cn_hinc $(=0.15 \%)$, chronic $(=$ $51.16 \%)$, alcoholicg $(=0.12 \%)$, and smokingg $(=0.93 \%)$ were higher than the $0.13 \%$ cutoff criterion, which indicated they were potential confounding variables.

Supplementary Table 8 showed that the change-inestimates of smokingg $(=0.08 \%)$ were lower than the $0.09 \%$ cutoff criterion, which indicated they were covariates. However, the change-in-estimates of age $(=0.86 \%)$, sex $(=0.38 \%)$, maritaln $(=0.34 \%)$, religion $(=0.13 \%)$, urbruraln $(=81.15 \%)$, educyrs $(=17.45 \%)$, bmi $(=9.42 \%)$, employment $(=0.29 \%)$, hhdnum $(=1.71 \%)$, cn_hinc (= $0.9726 \%)$, chronic $(=18.24 \%)$, and alcoholicg $(=0.17 \%)$ were higher than the $0.12 \%$ cutoff criterion, which indicated they were potential confounding variables.

Supplementary Table 9 showed that the change-inestimates of religion $(=0.08 \%)$, hhdnum $(=0.08 \%)$, and alcoholicg $(=0.02 \%)$ were lower than the $0.09 \%$ cutoff criterion, which indicated they were covariates. However, the change-in-estimates of age $(=15.87 \%)$, sex $(=0.65 \%)$, maritaln $(=0.58 \%)$, urbruraln $(=60.37 \%)$, educyrs $(=$ $2.16 \%)$, bmi $(=0.31 \%)$, employment $(=1.96 \%)$, cn_hinc (= $0.38 \%)$, chronic $(=6.87 \%)$, and smokingg $(=2.89 \%)$ were higher than the $0.30 \%$ cutoff criterion, which indicated they were potential confounding variables.

Supplementary Table 10 showed that the change-inestimates of religion $(=0.03 \%)$ and alcoholicg $(=0.01 \%)$ were lower than the $0.09 \%$ cutoff criterion, which indicated they were covariates. However, the change-inestimates of age $(=2.44 \%)$, sex $(=1.09 \%)$, maritaln $(=$ $0.61 \%)$, urbruraln $(=43.09 \%)$, educyrs $(=4.14 \%)$, bmi $(=$ $0.26 \%)$, employment $(=0.78 \%)$, hhdnum $(=0.59 \%)$, cn hinc $(=2.27 \%)$, chronic $(=2.36 \%)$, and smoking $(=0.21 \%)$ were higher than the $0.26 \%$ cutoff criterion, which indicated they were potential confounding variables.

Supplementary Table 11 showed that the change-inestimates of employment $(=0.04 \%)$ and hhdnum (= $0.03 \%$ ) were lower than the $0.09 \%$ cutoff criterion, which indicated they were covariates. However, the change-inestimates of age $(=38.25 \%)$, sex $(=0.37 \%)$, maritaln $(=$ $2.07 \%)$, religion $(=0.16 \%)$, urbruraln $(=87.79 \%)$, educyrs $(=6.81 \%)$, bmi $(=0.60 \%)$, cn_hinc $(=0.27 \%)$, chronic (=
$23.20 \%)$, alcoholicg $(=0.41 \%)$, and smokingg $(=4.23 \%)$ were higher than the $0.15 \%$ cutoff criterion, which indicated they were potential confounding variables.

Supplementary Table 12 showed that the change-inestimates of smokingg $(=0.01 \%)$ was lower than the $0.09 \%$ cutoff criterion, which indicated they were covariates. However, the change-in-estimates of age $(=4.98 \%)$, sex $(=1.01 \%)$, maritaln $(=1.41 \%)$, religion $(=0.15 \%)$, urbruraln $(=61.63 \%)$, educyrs $(=8.03 \%)$, bmi $(=0.38 \%)$, employment $(=0.21 \%)$, hhdnum $(=0.30 \%)$, cn_hinc (= $1.8066 \%)$, chronic $(=6.77 \%)$, and alcoholicg $(=0.18 \%)$ were higher than the $0.14 \%$ cutoff criterion, which indicated they were potential confounding variables.

Table 4 showed the associations between two cooccurring perceived pollution and QoL. The perceived air $\times$ noise pollution had significant associations with PCS scores $(\beta=13.880,95 \% \mathrm{CI}: 11.037,16.722)$ and MCS scores ( $\beta=31.126,95 \%$ CI: 29.218, 33.034). The perceived air $\times$ water pollution had significant associations with PCS scores $(\beta=9.352,95 \% \mathrm{CI}: 6.529,12.175)$ and MCS scores $(\beta=30.913, \quad 95 \% \mathrm{CI}: \quad 28.798,33.028)$. The perceived noise $\times$ water pollution had significant associations with PCS scores $(\beta=11.051,95 \%$ CI: 7.966,14.135) and MCS scores $(\beta=30.768,95 \%$ CI: $28.595,32.942)$.

Alcoholic had significant associations with PCS scores $(\beta=13.638,95 \%$ CI: 11.503, 15.773) and MCS scores $(\beta=$ 39.970, 95\%CI: 38.746, 41.194) in the population with perceived air $\times$ water pollution. Hhdnum had significant associations with PCS scores $(\beta=12.566,95 \%$ CI: 11.821 , $13.310)$ in the population with perceived air $\times$ noise pollution, PCS scores $(\beta=11.505,95 \% \mathrm{CI}$ : $10.692,12.318)$ in the population with perceived air $\times$ water pollution, and PCS scores $(\beta=11.951,95 \%$ CI: 11.116, 12.786) in the population with perceived noisexwater pollution. Religion had significant associations with PCS scores $(\beta=$ 3.377, 95\%CI: $-0.256,7.009)$ and MCS scores $(\beta=$ 32.172, 95\%CI: 29.580, 34.765). Smokingg had significant associations with MCS scores $(\beta=44.011,95 \% \mathrm{CI}$ : $43.210,44.813)$ in the population with perceived air $\times$ noise pollution and MCS scores $(\beta=45.298$, 95\%CI: $44.551,46.045)$ in the population with perceived noisexwater pollution. Employment had significant associations with PCS scores $(\beta=9.127,95 \% \mathrm{CI}$ : $6.640,11.613)$ in the population with perceived noisexwater pollution.

\section{Three co-occurring perceived environmental pollution}

Supplementary Table 13 showed that the change-inestimates of hhdnum (=0.00\%) was lower than the $0.09 \%$ cutoff criterion, which indicated they were covariates. However, the change-in-estimates of age $(=9.17 \%)$, sex $(=1.20 \%)$, maritaln $(=2.04 \%)$, religion $(=0.1620 \%)$, urbruraln $(=2.49 \%)$, educyrs $(=196.99 \%)$, bmi $(=0.45 \%)$, employment $(=1.96 \%)$, cn_hinc $(=0.29 \%)$, chronic (= $39.37 \%)$, alcoholicg $(=0.23 \%)$, and smokingg $(=5.99 \%)$ 
Table 4 Associations between two co-occurring pollution and PCS and MCS scores, Coefficients (95\%Cl)

\begin{tabular}{|c|c|c|c|c|c|c|}
\hline & \multicolumn{2}{|l|}{ Airxnoise } & \multicolumn{2}{|l|}{ Airxwater } & \multicolumn{2}{|l|}{ Noisexwater } \\
\hline & PCS scores & MCS scores & PCS scores & MCS scores & PCS scores & MCS scores \\
\hline Airxnoise & $\begin{array}{l}13.880^{* * *} \\
(11.037,16.722)\end{array}$ & $\begin{array}{l}31.126^{* * *} \\
(29.218,33.034)\end{array}$ & & & & \\
\hline Air $\times$ water & & & $\begin{array}{l}9.352^{* * *} \\
(6.529,12.175)\end{array}$ & $\begin{array}{l}30.913^{* * * *} \\
(28.798,33.028)\end{array}$ & & \\
\hline Noisexwater & & & & & $\begin{array}{l}11.051^{* * *} \\
(7.966,14.135)\end{array}$ & $\begin{array}{l}30.768^{* * *} \\
(28.595,32.942)\end{array}$ \\
\hline Alcoholicg & & & $\begin{array}{l}13.638^{* * *} \\
(11.503,15.773)\end{array}$ & $\begin{array}{l}39.970^{* * *} \\
(38.746,41.194)\end{array}$ & & \\
\hline Hhdnum & $\begin{array}{l}12.566^{* * *} \\
(11.821,13.310)\end{array}$ & & $\begin{array}{l}11.505^{* * *} \\
(10.692,12.318)\end{array}$ & & $\begin{array}{l}11.951^{* * *} \\
(11.116,12.786)\end{array}$ & \\
\hline Religion & & & $\begin{array}{l}3.377^{*} \\
(-0.256,7.009)\end{array}$ & $\begin{array}{l}32.172^{* * *} \\
(29.580,34.765)\end{array}$ & & \\
\hline Smokingg & & $\begin{array}{l}44.011^{* * *} \\
(43.210,44.813)\end{array}$ & & & & $\begin{array}{l}45.298^{* * *} \\
(44.551,46.045)\end{array}$ \\
\hline Employment & & & & & $\begin{array}{l}9.127^{* * *} \\
(6.640,11.613)\end{array}$ & \\
\hline R-squared & 0.789 & 0.438 & 0.801 & 0.435 & 0.793 & 0.426 \\
\hline Number of observation & 3774 & 3751 & 3745 & 3746 & 3726 & 3747 \\
\hline
\end{tabular}

Note: ${ }^{* *}, * *$ and ${ }^{*}$ indicates 1,5 and $10 \%$ significance level, respectively. Alcoholicg $=$ frequencies of alcoholic drinks. Hhdnum $=$ number of household members. Religion $=$ religious status. Smokingg $=$ frequencies of smoking

were higher than the $0.16 \%$ cutoff criterion, which indicated they were potential confounding variables.

Supplementary Table 14 showed that the change-inestimates of hhdnum $(=0.03 \%)$ was lower than the $0.09 \%$ cutoff criterion, which indicated they were covariates. However, the change-in-estimates of age $(=3.72 \%)$, sex $(=1.14 \%)$, maritaln $(=1.50 \%)$, religion $(=0.27 \%)$, urbruraln $(=62.15 \%)$, educyrs $(=12.33 \%)$, bmi $(=0.41 \%)$, employment $(=0.56 \%)$, cn_hinc $(=1.90 \%)$, chronic $(=12.12 \%)$, alcoholicg $(=0.10 \%)$, and smokingg $(=0.40 \%)$ were higher than the $0.09 \%$ cutoff criterion, which indicated they were potential confounding variables.

Table 5 showed the associations between three cooccurring perceived pollution and QoL. The perceived air $\times$ noise $\times$ water pollution had significant associations with PCS scores $(\beta=12.119,95 \%$ CI: 8.578, 15.659) and MCS scores $(\beta=11.216,95 \%$ CI: 7.768, 14.665). Hhdnum had significant associations with PCS scores $(\beta=12.792$, 95\%CI: $12.056,13.528)$ and MCS scores $(\beta=12.396$, 95\%CI: 11.682, 13.109).

\section{Moderation analysis}

The results in the potential models 1,2 , and 3 did not reach statistical significance in the supplementary Figs. 136. Thus, the simple moderation relationships were rejected. When mediators were dichotomous, the models 6,7 , and 8 couldn't be used. Thus, one pollutant could not affect one of both PCS and MCS scores through another pollutant. The other potential models $9,21,22$, and 70 also had no significant mediating outcomes. The $p$-values of interactions were not more than 0.10 , the moderated mediation relationships were established. After biascorrected 95\% confidence interval (CI) with 1000 bootstrapping samples was calculated, Figs. 1 and 2 were identified the statistically according to model 10 .

In Fig. 1, perceived air pollution had a negative association with MCS scores $(\beta=-1.064, p=.0783)$. MCS scores had a positive association with PCS scores $(\beta=.197, p=.0000)$. Perceived water pollution had a negative association with PCS scores $(\beta=-1.742$, $p=.0048)$. Perceived air $\times$ water pollution had a positive association with PCS scores $(\beta=1.820, \quad p=.0426)$.

Table 5 Associations between three co-occurring pollution and PCS and MCS scores, Coefficients (95\%Cl)

\begin{tabular}{lll}
\hline & PCS scores & MCS scores \\
\hline Airxnoisexwater & $12.119^{* * *}(8.578,15.659)$ & $11.216^{* * *}(7.768,14.665)$ \\
Hhdnum & $12.792^{* * *}(12.056,13.528)$ & $12.396^{* * *}(11.682,13.109)$ \\
R-squared & 0.784 & 0.780 \\
Number of observation & 3770 & 3770 \\
\hline
\end{tabular}

Note: ***,** and ${ }^{*}$ indicates 1,5 and $10 \%$ significance level, respectively. Hhdnum $=$ number of household members 


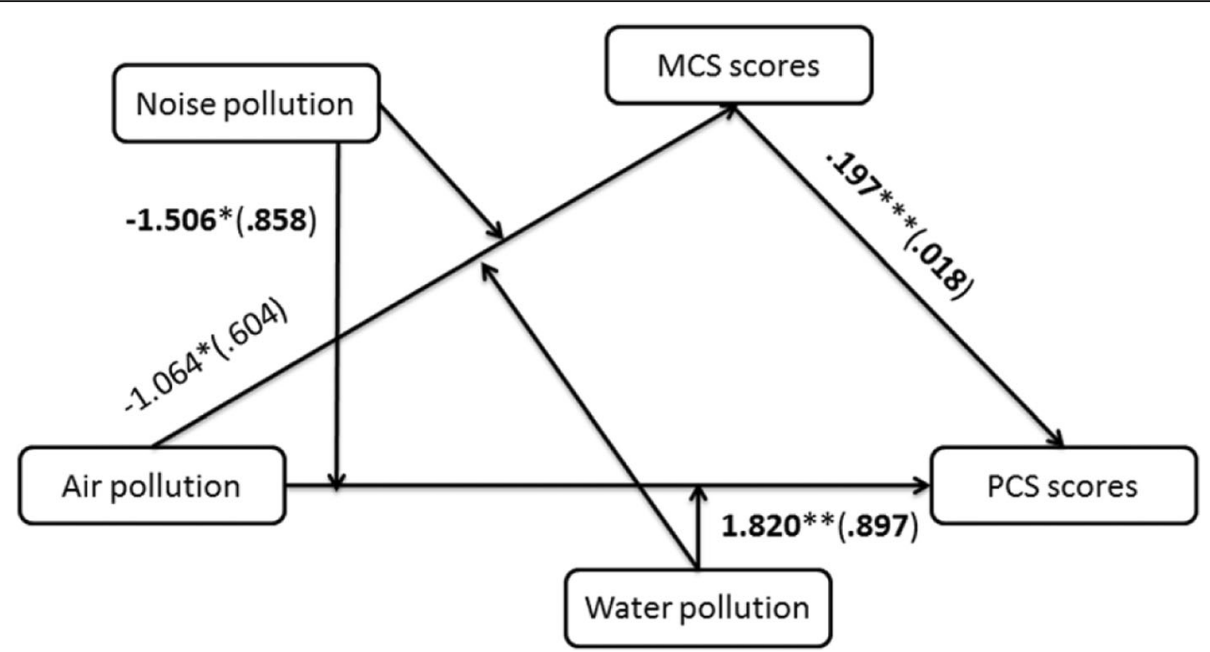

Fig. 1 Moderated mediation model controlling for employment, bmi, hhdnum, religion, alcoholicg, and smokingg ( $N=3697)$. Statistically significant path coefficients were indicated with asterisks $\left(*^{* *}, 1 \%\right.$; ${ }^{*}, 5 \%$; and $\left.{ }^{*}, 10 \%\right)$

Perceived noise pollution had a positive association with PCS scores $(\beta=1.643, p=.0028)$. Perceived air $\times$ noise pollution had a negative association with PCS scores $(\beta=-1.506, p=.0794)$. Thus, there were no moderating effects and mediating effects of perceived air pollution on PCS scores.

In Fig. 2, perceived water pollution had a negative association with PCS scores $(\beta=-1.906, p=.0024)$. Perceived air $\times$ water pollution had a positive association with PCS scores $(\beta=1.957, p=.0318)$. Perceived noise pollution had a positive association with PCS scores ( $\beta=1.545, p=.0057)$. PCS scores had a positive association with MCS scores $(\beta=.158, p=.0000)$. Perceived air pollution had a negative association with MCS scores $(\beta=-1.143, p=.0548)$. Perceived air $\times$ noise pollution had a positive association with MCS scores $(\beta=1.275$, $p=.0978)$. Thus, there were no moderating effects and mediating effects of perceived air pollution on MCS scores.

\section{Discussion}

On the novel use of the SF-12 in a Chinese adult population, this study reported the associations between perceived environmental pollution and PCS and MCS scores. Only considering air pollution, air pollution and water pollution had significant associations with PCS and MCS scores, respectively. Noise pollution had significant associations with PCS scores. The air $\times$ noise pollution, air $\times$ water pollution, and noisexwater pollution had significant associations with PCS and MCS scores. The air $\times$ noise $\times$ water pollution had significant associations with PCS and MCS scores. Thus, it could be

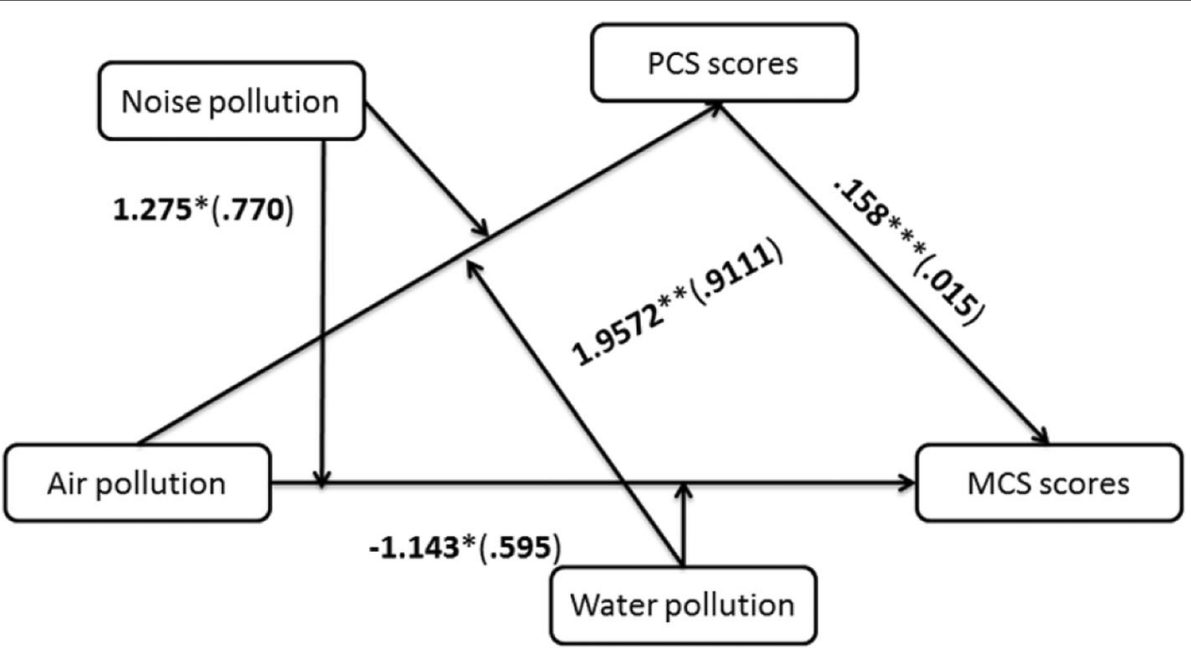

Fig. 2 Moderated mediation model controlling for employment, bmi, hhdnum, religion, alcoholicg, and smokingg ( $N=3697)$. Statistically significant path coefficients were indicated with asterisks (***, 1\%; **, 5\%; and *, 10\%) 
speculated that number of co-occurring perceived environmental pollution could increase PCS and MCS scores. Furthermore, this study highlighted the importance of socioeconomic factors (number of household members, religion, and employment) and lifestyle variables (body mass index, frequencies of smoking, and frequencies of alcoholic drinks) in the increasing PCS and MCS scores in this population.

It might be surprised that perceived environmental pollution could increase HRQoL on the basis of PCS and MCS scores in the general Chinese population. Especially, the outcomes in this study could be opposite of an early study that air pollution might worsen mental health in China [48]. An early study also reported there might be a link between exposure to air pollution and psychiatric disorders in children and adolescents [49]. In modern China, the common population often was cultivated with respect to cleanness and hygiene and educated the knowledge of environmental prevention. In order to fight against environmental degradation, China government controlled high pollution industries, demolished higher-polluting iron and steel works, and carried on strict policies on the discharge of harmful chemicals and compounds. Simultaneously, health authorities in China made efforts to reduce the long-term threats posed by environmental pollution. Chinese individuals were thus aware of the seriousness of environmental pollution and health risk in the neighborhood. Due to cognitive stress, they possibly overestimate their health risks of environmental pollution and carried out preventive countermeasures.

Prior studies in international circle could construct an evident chain to explain the fact in China's setting. Several population-based questionnaire studies indicated perceived pollution and health risk perception played important roles in understanding and predicting environmentally induced symptoms and diseases [50, 51]. A mail-based questionnaire study concluded the capacity to process and perceived environmental health threats in a positive manner might be a valuable human ability positively influencing personal satisfaction and wellbeing [52]. Perceived risk of environmental threats often translated into psychological stress with a wide range of effects on health and well-being.

The findings here were in line with the prior worldwide research that documented the relationship between socioeconomic factors and lifestyle variables and HRQoL globally. Regarding number of household members, several studies reported that living arrangement was associated with higher risk of poor HRQoL [53, 54]. Considering the role of religion, Chen and Williams (2016) argued that the private and subjective dimension of religion mattered for well-being in China [55]. A growing body of peer- reviewed articles found that religious involvement was associated with QoL in old age [56], amongst older Ethiopians [57], and in patients with schizophrenia in Latin America [58].

With respect to employment status, the findings in this study were in line with the results in several early [59-62]. Clinically, employment was associated with QoL in multiple sclerosis patients [63], HRQoL among people with multiple sclerosis [64], HRQoL among people with schizophrenia [65], and HRQoL among Hungarian Hodgkin lymphoma survivors [66]. Thus, the associations could be generalized.

Regarding the association between BMI and HRQoL, the findings in this study were not in agreement with inverse association between BMI and physical HRQoL and positive association between BMI and mental HRQoL [67], inverse U-shaped association scores [68], and complex association [69]. Regarding the association between smoking and HRQoL, the findings in this study were not in agreement with inverse association [70-72]. Regarding the association between alcohol drinking and HRQoL, the findings in this study were in line with positive association between alcohol drinking and physical HRQoL [73], positive association between alcohol consumption and higher HRQoL in female [74]. The findings in this study were not in line with negative associations [75] in the early studies.

To my best knowledge, this is the first study to report the concurrent pollution rather than single pollution had associations with poor HRQoL. But, this study was not in agreement with the results from the prior studies due to different research designs. For example, air pollution and noise pollution had impact on health independently in North Island of New Zealand [76]. But, this study integrated two or three pollution into one. Similarly, this study split HRQoL into PCS and MCS instead of eight subdomains. Still, literature [76] was taken as an example. Without interactions between air pollution annoyance and noise annoyance, literature [76] indicated that air pollution annoyance and noise annoyance effectively predicted variability in the different HRQoL domains. This study also discorded with previous population-based studies conducted in other countries, in which HRQoL was considered as a covariant to predict perceptions of environmental pollution. Kamimura, et al. (2017) also used the East Asia data to conclude the integral HRQoL rather than sectional HRQoL was associated with levels of perceived environmental pollution [77]. Thus, this study enriched the knowledge of the relationship between pollution and HRQoL.

Exposure to environmental pollution was associated with the growing morbidity and mortality worldwide. Thus, some academic fruits pointed to the direction of governmental conceiving in China [78-80]. Due to high 
level of perceived environmental health threats, subjective PCS and MCS scores were high, and more preparedness possibly had occurred at the individual or household levels. This report had potential to inform the development of related policies and risk communication strategies in China. Thus, new immediate solutions should be conceived to curb the pervasive environmental problem.

The present study had several limitations. First, this study was conducted with missing data and without conducting imputation. Although the observations with missing data were a relatively small proportion of the eligible sample, the research outcomes were not reflected correct in the whole. Second, subjective assessments were adopted in a cross-sectional survey. Thus, due to lack of data on availability and accessibility of natural environments in the neighborhood, the role of beforeafter environmental epidemiology in the HRQoL could not be discovered. The subjective data also had limited abilities to allow causal study to be conducted among variables. Finally, since the some variables were controlled for, the current data framework might not reflect the demographic factors and HRQoL completely. Future analyses should make efforts to fill in the gaps.

\section{Conclusions}

In conclusion, sociodemographic characteristics, lifestyle, and perceived environmental pollution were associated with PCS and MCS scores. The perceived single and cooccurring environmental pollution had significant associations with PCS and MCS scores in Chinese adult population. But, there were no moderating and mediating relationships among the perceived environmental pollutions. Further studies were to explore the causal effects of environmental pollution on PCS and MCS scores.

\section{Supplementary information}

Supplementary information accompanies this paper at https://doi.org/10. 1186/s12955-020-01442-9.

Additional file 1: Supplementary Table 1. Change-in-estimate for PCS scores with possible confounding factors. Supplementary Table 2. Change-in-estimate for MCS scores with possible confounding factors. Supplementary Table 3. Change-in-estimate for PCS scores with possible confounding factors. Supplementary Table 4. Change-in-estimate for MCS scores with possible confounding factors. Supplementary Table 5. Change-in-estimate for PCS scores with possible confounding factors. Supplementary Table 6. Change-in-estimate for MCS scores with possible confounding factors. Supplementary Table 7. Change-inestimate for PCS scores with possible confounding factors. Supplementary Table 8. Change-in-estimate for MCS scores with possible confounding factors. Supplementary Table 9. Change-in-estimate for PCS scores with possible confounding factors. Supplementary Table 10. Change-in-estimate for MCS scores with possible confounding factors. Supplementary Table 11. Change-in-estimate for PCS scores with possible confounding factors. Supplementary Table 12. Change-inestimate for MCS scores with possible confounding factors. Supplementary Table 13. Change-in-estimate for PCS scores with possible confounding factors. Supplementary Table 14. Change-in-estimate for MCS scores with possible confounding factors.

Additional file $\mathbf{2}$ Potential conceptual diagrams.

Additional file $\mathbf{3}$ Statistical outcomes of potential conceptual diagrams.

\section{Abbreviations}

HRQoL: Health-related quality of life; MCS: Mental component summary; PCS: Physical component summary; PF: Physical functioning; RP: Role physical; BP: Bodily pain; GH: General health; VT: Vitality; SF: Functioning; RE: Role Emotional; MH: Mental health; BMI: Body mass index; AOR: Adjusted odds ratio

\section{Acknowledgements}

I wish to acknowledge the very helpful comments of anonymous reviewers of the original submission.

\section{Author's contributions}

$\mathrm{BH}$ carried out all the research process, including designing the study and performing the statistical analysis. Also, $\mathrm{BH}$ drafted, read and approved the final manuscript.

\section{Funding}

This project was funded by the Mechanism of Bidirectional Flow of Rural Population in Henan Province (in Chinese: 河南农村人口双向流动的机理 研究; Project number: 2019-ZDJH-413) from 2019 general projects of Humanities and Social Sciences in the Henan Provincial Department of Education (in Chinese: 河南省教育 厅2019年度人文社会科学研究一般项

目指导性计划), Construction study and practice of ideological and political teaching in the course of China Geography (in Chinese: 《中国地理》课程思 政建设研究与实践; Project number: 407) from Research and Practice Project of Higher Education \& Pedagogy Reform in Henan Province in 2019 (in Chinese: 2019年度河南省高等教育教学改革研究与实践项目), and Design and Implementation of Integrated Service Mode of "Environmental Protection Housekeeper" in Zhangpan Chemical Park of Xuchang City (in Chinese: 许昌市张潘化工园区“环保管家”综合服务模式设计与实施; Project number: 10) from University-enterprise cooperation project organized by Xuchang City Science and Technology Bureau, 2017 (in Chinese: 2017 2017年许昌市科技局校地合作专项立项项目)

Availability of data and materials http://www.eassda.org/

Ethics approval and consent to participate

This study used publicly available dataset. So, it is unnecessary to be proved by ethics committee.

Consent for publication

Not applicable.

Competing interests

The author declares that he has no competing interests.

\section{Author details}

${ }^{1}$ International Issues Center, Xuchang University, Xuchang, Henan, China.

${ }^{2}$ Family Issues Center, Xuchang University, Xuchang, Henan, China. ${ }^{3}$ Xuchang Urban Water Pollution Control and Ecological Restoration Engineering Technology Research Center, Xuchang University, Xuchang, China. ${ }^{4}$ College of Urban and Environmental Sciences, Xuchang University, Xuchang, China.

Received: 19 February 2020 Accepted: 5 June 2020

Published online: 23 June 2020

References

1. Bose S, Romero K, Psoter KJ, Curriero FC, Chen C, Johnson CM, Kaji D, Breysse PN, Williams DL, Ramanathan M, Checkley W, Hansel NN. Association of traffic air pollution and rhinitis quality of life in Peruvian children with asthma. PLoS One. 2018;13(3):e0193910. https://doi.org/10. 1371/journal.pone.0193910 eCollection 2018.

2. Pirozzi CS, Mendoza DL, Xu Y, Zhang Y, Scholand MB, Baughman RP. ShortTerm Particulate Air Pollution Exposure is Associated with Increased Severity 
of Respiratory and Quality of Life Symptoms in Patients with Fibrotic Sarcoidosis. Int J Environ Res Public Health. 2018;15(6):E1077. https://doi.org/ 10.3390/ijerph15061077.

3. Nakao M, Ishihara Y, Kim CH, Hyun IG. The impact of air pollution, including Asian sand dust, on respiratory symptoms and health-related quality of life in outpatients with chronic respiratory disease in Korea: a panel study. J Prev Med Public Health. 2018;51(3):130-9. https://doi.org/10.3961/jpmph.18. 021 Epub 2018 May 9.

4. Yung J, Osahan S, Friedman SM, Li J, Cone JE. Air Pollution/lrritants, Asthma Control, and Health-Related Quality of Life among 9/11-Exposed Individuals with Asthma. Int J Environ Res Public Health. 2019;16(11):E1924. https://doi. org/10.3390/ijerph16111924.

5. Kim K-N, Lee H, Kim JH, Jung K, Lim Y-H, Hong Y-C. Physical activity- and alcohol-dependent association between air pollution exposure and elevated liver enzyme levels: an elderly panel study. J Prev Med Public Health. 2015; 48(3):151-69.

6. Genc $\mathrm{S}$, Zadeoglulari Z, Fuss SH, Genc K. The adverse effects of air pollution on the nervous system. J Toxicol. 2012;2012:782462.

7. Pun VC, Manjourides J, Suh H. Association of Ambient air Pollution with depressive and anxiety symptoms in older adults: results from the NSHAP study. Environ Health Perspect. 2017;125(3):342-8.

8. Egondi T, Kyobutungi C, Ng N, Muindi K, Oti S, van de Vijver S, Ettarh R, Rocklöv J. Community perceptions of air pollution and related health risks in Nairobi slums. Int J Environ Res Public Health. 2013;10(10):4851-68. https://doi.org/10.3390/ijerph10104851.

9. Mannucci PM, Franchini M. Health effects of ambient air pollution in developing countries. Int J Environ Res Public Health. 2017;14(9):1048.

10. Nakao M, Yamauchi K, Ishihara Y, Omori H, Ichinnorov D, Solongo B. Effects of air pollution and seasons on health-related quality of life of Mongolian adults living in Ulaanbaatar: cross-sectional studies. BMC Public Health. 2017; 17:594.

11. Nakao M, Yamauchi K, Ishihara Y, Solongo B, Ichinnorov D. Effects of air pollution and seasonality on the respiratory symptoms and health-related quality of life (HR-QoL) of outpatients with chronic respiratory disease in Ulaanbaatar: pilot study for the comparison of the cold and warm seasons. SpringerPlus. 2016;5(1):1817.

12. Weuve J, Kaufman JD, Szpiro AA, et al. Exposure to traffic-related air pollution in relation to progression in physical disability among older adults. Environ Health Perspect. 2016;124(7):1000-8.

13. Stieb DM, Shutt R, Kauri L, et al. Cardio-respiratory effects of air pollution in a panel study of outdoor physical activity and health in rural older adults. J Occup Environ Med. 2017;59(4):356-64.

14. Roberts JD, Voss JD, Knight B. The Association of Ambient air Pollution and Physical Inactivity in the United States. PLoS One. 2014;9(3):e90143.

15. Li F, Liu Y, Lü J, Liang L, Harmer P. Ambient air pollution in China poses a multifaceted health threat to outdoor physical activity. J Epidemiol Community Health. 2015;69(3):201-4.

16. Lu Y, Song S, Wang R, Liu Z, Meng J, Sweetman AJ, Jenkins A, Ferrier RC, Li $\mathrm{H}$, Luo W, Wang T. Impacts of soil and water pollution on food safety and health risks in China. Environ Int. 2015;77:5-15.

17. Yang X, Duan J, Wang L, Li W, Guan J, Beecham S, Mulcahy D. Heavy metal pollution and health risk assessment in the Wei River in China. Environ Monit Assess. 2015;187(3):111. https://doi.org/10.1007/s10661-014-4202-y.

18. Wang $Q$, Yang Z. Industrial water pollution, water environment treatment, and health risks in China. Environ Pollut. 2016;218:358-65. https://doi.org/10. 1016/j.envpol.2016.07.011 Epub 2016 Jul 18.

19. Levy K. Does Poor Water Quality Cause Diarrheal Disease? Am J Trop Med Hyg. 2015;93(5):899-900.

20. Queiroz AC, Cardoso LS, Heller L, Cairncross S. Integrating surveillance data on water-related diseases and drinking-water quality; action-research in a Brazilian municipality. J Water Health. 2015;13(4):1048-54.

21. Ren H, Wan X, Yang F, Shi X, Xu J, Zhuang D, Yang G. Association between changing mortality of digestive tract cancers and water pollution: a case study in the Huai River basin, China. Int J Environ Res Public Health. 2015;12(1):214-26

22. Al-Hammad BA, Abd El-Salam MM. Monitoring water pollution levels in Wadi Hanifa, Riyadh, Saudi Arabia and its public health implications. Bull Environ Contam Toxicol. 2017;98(4):525-33. https://doi.org/10.1007/s00128017-2048-z Epub 2017 Feb 18.

23. Dwivedi S, Mishra S, Tripathi RD. Ganga water pollution: a potential health threat to inhabitants of ganga basin. Environ Int. 2018;117:327-38. https:// doi.org/10.1016/j.envint.2018.05.015 Epub 2018 May 18. Review.
24. Hasan MK, Shahriar A, Jim KU. Water pollution in Bangladesh and its impact on public health. Heliyon. 2019;5(8):e2145 101016/jheliyon2019e02145 eCollection 2019 Aug. Review.

25. Xu C, Xing D, Wang J, Xiao G. The lag effect of water pollution on the mortality rate for esophageal cancer in a rapidly industrialized region in China. Environ Sci Pollut Res Int. 2019;26(32):32852-8. https://doi.org/10. 1007/s11356-019-06408-z Epub 2019 Sep 9.

26. Ali A, Hussain RM, Dom NC, Md Rashid Rl. A profile of noise sensitivity on the health-related quality of life among young motorcyclists. Noise Health. 2018;20(93):53-9 doi: 10.4103/nah. NAH_14_17.

27. Shepherd D, Landon J, Kalloor M, Barker-Collo S, Starkey N, Jones K, Ameratunga S, Theadom A. BIONIC Research Group. The association between health-related quality of life and noise or light sensitivity in survivors of a mild traumatic brain injury. Qual Life Res. 2019. https://doi. org/10.1007/s11136-019-02346-y.

28. Clark C, Paunovic K. WHO environmental noise guidelines for the European region: a systematic review on environmental noise and quality of life, Wellbeing and Mental Health. Int J Environ Res Public Health. 2018;15:2400.

29. Buxton RT, McKenna MF, Mennitt D, Fristrup K, Crooks K, Angeloni L, Wittemyer G. Noise pollution is pervasive in U.S. protected areas. Science. 2017;356(6337):531-3.

30. Mohammadizadeh M, Ahmadi SH, Sekhavati E, Ahani-Jegar K. Noise pollution effect in flour factory on workers' hearing in Lamerd City. J Med Life. 2015;8(Spec Iss 3):208-11.

31. Noweir MH, Bafail AO, Jomoah IM. Noise pollution in metalwork and woodwork industries in the Kingdom of Saudi Arabia. Int J Occup Saf Ergon. 2014;20(4):661-70.

32. Monazzam MR, Karimi E, Abbaspour M, Nassiri P, Taghavi L. Spatial traffic noise pollution assessment - a case study. Int J Occup Med Environ Health. 2015;28(3):625-34.

33. Héritier H, Vienneau D, Frei P, Eze IC, Brink M, Probst-Hensch N, Röösli M. The association between road traffic noise exposure, annoyance and health-related quality of life (HRQOL). Int J Environ Res Public Health. 2014;11(12):12652-67.

34. Roswall N, Høgh V, Envold-Bidstrup P, Raaschou-Nielsen O, Ketzel M, Overvad K, Olsen A, Sørensen M. Residential exposure to traffic noise and health-related quality of life-a population-based study. PLoS One. 2015;10(3):e0120199.

35. Schreckenberg D, Meis M, Kahl C, Peschel C, Eikmann T. Aircraft noise and quality of life around Frankfurt airport. Int J Environ Res Public Health. 2010; 7(9):3382-405

36. de Paiva Vianna KM, Alves Cardoso MR, Rodrigues RM. Noise pollution and annoyance: an urban soundscapes study. Noise Health. 2015;17(76):125-33.

37. Tonne C, Halonen Jl, Beevers SD, Dajnak D, Gulliver J, Kelly FJ, Wilkinson P, Anderson HR. Long-term traffic air and noise pollution in relation to mortality and hospital readmission among myocardial infarction survivors. Int J Hyg Environ Health. 2016;219(1):72-8.

38. Gupta A, Gupta A, Jain K, Gupta S. Noise pollution and impact on children health. Indian J Pediatr. 2018:85(4):300-6.

39. Van de Werf F, McGuinness M, Gillespie S, Andriukaitis V. European commissioners petitioned for clean air and reduced noise pollution. Eur Heart J. 2015;36(47):3304-5.

40. Dritsaki M, Petrou S, Williams M, Lamb SE. An empirical evaluation of the SF-12, SF-6D, EQ-5D and Michigan hand outcome questionnaire in patients with rheumatoid arthritis of the hand. Health Qual Life Outcomes. 2017;15(1):20.

41. Conrad I, Matschinger H, Riedel-Heller S, von Gottberg C, Kilian R. The psychometric properties of the German version of the WHOQOL-OLD in the German population aged 60 and older. Health Qual Life Outcomes. 2014;12:105.

42. Silveira MF, Almeida JC, Freire RS, Haikal DS, Martins AE. Psychometric properties of the quality of life assessment instrument: 12-item health survey (SF-12). Cien Saude Colet. 2013;18(7):1923-31 Portuguese.

43. Kim SH, Jo MW, Ahn J, Ock M, Shin S, Park J. Assessment of psychometric properties of the Korean SF-12 V2 in the general population. BMC Public Health. 2014;14:1086. https://doi.org/10.1186/1471-2458-14-1086.

44. Lam CLK, Eileen YY, Gandek TB. The standard SF-12 was valid and equivalent for the Chinese, which would enable more Chinese to be included in clinical trials that measure HRQoL. Qual Life Res. 2005;14:539-47.

45. Niels Henrik Bruun, 2015.SF12: Stata module to validate sf12 input and calculate sf12 version $2 \mathrm{t}$ scores. Statistical Software Components S458125, Boston College Department of Economics, revised 2016.

46. Lee PH. Should we adjust for a confounder if empirical and theoretical criteria yield contradictory results? A simulation study. Sci Rep. 2014;4:6085. https://doi.org/10.1038/srep06085. 
47. Lee $\mathrm{PH}$. Is a cutoff of $10 \%$ appropriate for the change-in-estimate criterion of confounder identification? J Epidemiol. 2014;24:161-7. https://doi.org/10. 2188/jea.JE20130062.

48. Wang $R$, Xue D, Liu Y, Liu P, Chen H. The Relationship between Air Pollution and Depression in China: Is Neighbourhood Social Capital Protective?.Int J Environ Res Public Health. 2018;15 (6). pii: E1160. doi: https://doi.org/10. 3390/ijerph15061160.

49. Oudin A, Bråbäck L, Åström DO, Strömgren M, Forsberg B. Association between neighbourhood air pollution concentrations and dispensed medication for psychiatric disorders in a large longitudinal cohort of Swedish children and adolescents. BMJ Open. 2016;6(6):e010004.

50. Claeson AS, Lidén E, Nordin M, Nordin S. The role of perceived pollution and health risk perception in annoyance and health symptoms: a population-based study of odorous air pollution. Int Arch Occup Environ Health. 2013;86(3):367-74. https://doi.org/10.1007/s00420-012-0770-8 Epub 2012 Apr 18.

51. Orru K, Nordin S, Harzia H, Orru H. The role of perceived air pollution and health risk perception in health symptoms and disease: a population-based study combined with modelled levels of PM10. Int Arch Occup Environ Health. 2018;91(5):581-9. https://doi.org/10.1007/s00420-018-1303-x.

52. Bränström R, Brandberg $Y$. Health risk perception, optimistic bias, and personal satisfaction. Am J Health Behav. 201;34(2):197-205.

53. Wittenberg E, Ritter GA, Prosser LA. Evidence of spillover of illness among household members: EQ-5D scores from a US sample. Med Decis Mak. 2013; 33(2):235-43. https:/doi.org/10.1177/0272989X12464434 Epub 2012 Oct 25.

54. Gjelsvik A, Dumont DM, Nunn A, Rosen DL. Adverse childhood events: incarceration of household members and health-related quality of life in adulthood. J Health Care Poor Underserved. 2014;25(3):1169-82. https://doi. org/10.1353/hpu.2014.0112

55. Chen $Y$, Williams M. Subjective well-being in the new China: religion, social capital, and social status. Br J Sociol. 2016;67(4):719-46. https://doi.org/10. 1111/1468-4446.12232

56. Idler EL, McLaughlin J, Kasl S. Religion and the quality of life in the last year of life. J Gerontol B Psychol Sci Soc Sci. 2009;64(4):528-37. https://doi.org/10. 1093/geronb/gbp028 Epub 2009 May 12.

57. Hamren K, Chungkham HS, Hyde M. Religion, spirituality, social support and quality of life: measurement and predictors CASP-12(v2) amongst older Ethiopians living in Addis Ababa. Aging Ment Health. 2015;19(7):610-21. https://doi.org/10.1080/13607863.2014.952709 Epub 2014 Aug 30.

58. Caqueo-Urízar A, Urzúa A, Boyer L, Williams DR. Religion involvement and quality of life in patients with schizophrenia in Latin America. Soc Psychiatry Psychiatr Epidemiol. 2016;51(4):521-8. https://doi.org/10.1007/s00127-0151156-5 Epub 2015 Nov 27

59. van Rijn RM, Carlier BE, Schuring M, Burdorf A. Work as treatment? The effectiveness of re-employment programmes for unemployed persons with severe mental health problems on health and quality of life: a systematic review and meta-analysis. Occup Environ Med. 2016;73(4):275-9. https://doi. org/10.1136/oemed-2015-103121 Epub 2016 Jan 6. Review.

60. Min D, Cho E. Patterns in quality of life according to employment among the older adults: the Korean longitudinal study of aging (2008-2014). BMC Public Health. 2018;18(1):379. https://doi.org/10.1186/s12889-018-5296-X.

61. Chen X, Yu B, Gong J, Wang P, Elliott AL. Social capital associated with quality of life mediated by employment experiences: evidence from a random sample of rural-to-urban migrants in China. Soc Indic Res. 2018; 139(1):327-46. https://doi.org/10.1007/s11205-017-1617-1 Epub 2017 Apr 6.

62. Marck CH, Aitken Z, Simpson S Jr, Weiland TJ, Kavanagh A, Jelinek GA. Predictors of change in employment status and associations with quality of life: a prospective international study of people with multiple sclerosis. J Occup Rehabil. 2019. https://doi.org/10.1007/s10926-019-09850-5 [Epub ahead of print].

63. Šabanagić-Hajrić S, Alajbegović A. Impacts of education level and employment status on health-related quality of life in multiple sclerosis patients. Med Glas (Zenica). 2015;12(1):61-7.

64. Chiu CY, Chan F, Edward Sharp S, Dutta A, Hartman E, Bezyak J. Employment as a health promotion intervention for persons with multiple sclerosis. Work. 2015;52(4):749-56. https://doi.org/10.3233/WOR-152202.

65. Bouwmans C, de Sonneville C, Mulder CL, Hakkaart-van RL. Employment and the associated impact on quality of life in people diagnosed with schizophrenia. Neuropsychiatr Dis Treat. 2015;11:2125-42. https://doi.org/10. 2147/NDT.S83546 eCollection 2015. Review.
66. Magyari F, Kósa K, Berecz R, Illés A, Miltényi Z, Simon Z, Illés Á. Employment status and health related quality of life among Hodgkin-lymphoma survivors'- results based on data from a major treatment center in Hungary. Health Qual Life Outcomes. 2017;15(1):180. https://doi.org/10.1186/s12955017-0758-x.

67. Müller-Nordhorn J, Muckelbauer R, Englert H, et al. Longitudinal association between body mass index and health-related quality of life. PLoS One. 2014;9(3):e93071. Published 2014 Mar 26. https://doi.org/10.1371/journal. pone.0093071.

68. $X u$ Y, Zhou Z, Li Y, et al. Exploring the nonlinear relationship between body mass index and health-related quality of life among adults: a cross-sectional study in Shaanxi Province, China. Health Qual Life Outcomes. 2015;13:153. Published 2015 Sep 23. https://doi.org/10.1186/s12955-015-0347-9.

69. Zhang J, Xu L, Li J, et al. Gender differences in the association between body mass index and health-related quality of life among adults:a crosssectional study in Shandong, China. BMC Public Health. 2019;19(1):-1021. Published 2019 Jul 31. https://doi.org/10.1186/s12889-019-7351-7.

70. Chen PC, Kuo RN, Lai CK, Tsai ST, Lee YC. The relationship between smoking status and health-related quality of life among smokers who participated in a 1-year smoking cessation programme in Taiwan: a cohort study using the EQ-5D. BMJ Open. 2015;5(5):e007249. Published 2015 May 7. https://doi.org/ 10.1136/bmjopen-2014-007249.

71. Rezaei S, Matin BK, Karyani AK, Woldemichael A, Khosravi F, Khosravipour M, Rezaeian S. Impact of Smoking on Health-Related Quality of Life: A General Population Survey in West Iran. Asian Pac J Cancer Prev. 2017;18(11):317985. https://doi.org/10.22034/APJCP.2017.18.11.3179.

72. Rezaei S, Karami Matin B, Kazemi Karyani A, Woldemichael A, Khosravi F, Khosravipour M, Rezaeian S. Impact of smoking on health-related quality of life: a general population survey in West Iran. Asian Pac J Cancer Prev. 2017; 18(11):3179-85.

73. Ortolá R, García-Esquinas E, Galán I, Rodríguez-Artalejo F. Patterns of alcohol consumption and health-related quality of life in older adults. Drug Alcohol Depend. 2016;159:166-73. https://doi.org/10.1016/j.drugalcdep.2015.12.012 Epub 2015 Dec 24.

74. Bergman S, Symeonidou S, Andersson ML, Söderlin MK. BARFOT study group. Alcohol consumption is associated with lower self-reported disease activity and better health-related quality of life in female rheumatoid arthritis patients in Sweden: data from BARFOT, a multicenter study on early RA. BMC Musculoskelet Disord. 2013;14:218. https://doi.org/10.1186/14712474-14-218.

75. Imtiaz S, Loheswaran G, Le Foll B, Rehm J. Longitudinal alcohol consumption patterns and health-related quality of life: results from the National Epidemiologic Survey on alcohol and related conditions. Drug Alcohol Rev. 2018;37(1):48-55. https://doi.org/10.1111/dar.12503 Epub 2017 Mar 14.

76. Shepherd D, Dirks K, Welch D, McBride D, Landon J. The covariance between air pollution annoyance and noise annoyance, and its relationship with health-related quality of life. Int J Environ Res Public Health. 2016;13(8): 792.

77. Kamimura A, Armenta B, Nourian M, Assasnik N, Nourian K, Chernenko A. Perceived environmental pollution and its impact on health in China, Japan, and South Korea. J Prev Med Public Health. 2017;50(3):188-94.

78. Huang L, Li J, He R, Rao C, van der Kuijp TJ, Bi J. Quantitative analysis of health risk perception, exposure levels, and willingness to pay/accept of PM2.5 during the 2014 Nanjing youth Olympic games. Environ Sci Technol. 2018;52(23):13824-33. https://doi.org/10.1021/acs.est.8b01634 Epub 2018 Nov 13.

79. Wang S, Jiang J, Zhou Y, Li J, Zhao D, Lin S. Climate-change information, health-risk perception and residents' environmental complaint behavior: an empirical study in China. Environ Geochem Health. 2019. https://doi.org/10. 1007/s10653-018-0235-4 [Epub ahead of print].

80. Yang T. Association between perceived environmental pollution and health among urban and rural residents-a Chinese national study. BMC Public Health. 2020;20:194. https://doi.org/10.1186/s12889-020-8204-0.

\section{Publisher's Note}

Springer Nature remains neutral with regard to jurisdictional claims in published maps and institutional affiliations. 